\title{
Eccentricity Effects on Acoustic Radiation from a Spherical Source Suspended Within a Thermoviscous Fluid Sphere
}

\author{
Seyyed M. Hasheminejad and Mahdi Azarpeyvand
}

\begin{abstract}
Acoustic radiation from a spherical source undergoing angularly periodic axisymmetric harmonic surface vibrations while eccentrically suspended within a thermoviscous fluid sphere, which is immersed in a viscous thermally conducting unbounded fluid medium, is analyzed in an exact fashion. The formulation uses the appropriate wave-harmonic field expansions along with the translational addition theorem for spherical wave functions and the relevant boundary conditions to develop a closed-form solution in form of infinite series. The analytical results are illustrated with a numerical example in which the vibrating source is eccentrically positioned within a chemical fluid sphere submerged in water. The modal acoustic radiation impedance load on the source and the radiated far-field pressure are evaluated and discussed for representative values of the parameters characterizing the system. The proposed model can lead to a better understanding of dynamic response of an underwater acoustic lens. It is equally applicable in miniature transducer analysis and design with applications in medical ultrasonics.
\end{abstract}

\section{INTRODUCTION}

\begin{abstract}
A NalyTiCal solutions of interior or exterior boundary A value problems in various fields such as in potential theory, acoustics, and electromagnetism are severely limited by the shape of boundaries. In particular, when multiple objects are present in a sound field, there is an acoustical interaction between the objects due to cross scattering. Many researchers have studied the mutual interaction between obstacles subjected to a primary acoustic field [1]-[14]. Acoustic interaction force between two fluid spheres in a plane acoustic wave field, including the firstand second-order scattered fields of the objects, is investigated in [1]. Likewise, the translational addition theorems for the spherical wave functions are used in [2] to study scattering of sound waves at arbitrary angles of incidence by two interacting impenetrable spherical objects in detail. Later on, the same researchers used the three-dimensional elasticity shell theory to study acoustic scattering at arbitrary incidence by two fluid-filled spherical elastic shells above their coincidence frequency [3]. They also considered acoustic scattering by a hard spherical body near a hard flat boundary [4], by a thin spherical shell near a free (pressure release) surface [5], [6], and by an ideal

Manuscript received June 15, 2002; accepted June 9, 2003.

The authors are with the Department of Mechanical Engineering, Iran University of Science and Technology, Narmak, Tehran 16844, Iran (e-mail: hashemi@iust.ac.ir).
\end{abstract}

air-bubble near the sea surface [7]. An exact formalism, which includes the symmetry conditions of the scatterer system, is developed in [8] with the aim of investigating acoustic scattering by two identical spheres. A shape perturbation method is used in [9], [10] to derive analytical expressions for the acoustic resonance frequency shifts in a hard- (soft-) walled spherical caused by introduction of an eccentric small inner sphere. An exact solution based on the classical modal series method for the problem of radiation loading on a spherical source freely suspended in a fluid-filled spherical cavity with a permeable boundary has been developed in [11]. The more closely related problem of acoustic radiation from a spherical source embedded eccentrically within an inviscid fluid sphere and vibrating with an arbitrary, axisymmetric, time-harmonic velocity distribution is analyzed in [12]. This configuration is proposed as an idealization of a spherical acoustic lens, with focal point inside the lens, when used as a sound projector. In more recent papers [13], [14], the latter analysis is generalized for a number of nonaxisymmetric spherical sources within the fluid sphere.

Investigations of sound propagation and scattering with allowance for various dissipation mechanisms, such as viscous and thermal losses and complicated boundary or scatterer models, have been reported in many papers [15], [16]. The most well known acoustic theory for heterogeneous systems is developed by Epstein and Carhart [17], Allegra and Hawley [18] (i.e., the so-called ECAH theory). The ECAH theory takes into account the four most important loss mechanisms: viscous, thermal, scattering, and intrinsic [15]. It can describe attenuation for a monodisperse system of spherical particles and is principally valid for dilute systems. The ECAH method is extended to scattering by a free thermoelastic sphere submerged in a thermoviscous fluid in [19]. Likewise, acoustic scattering from a spherical shell, including thermoviscous effects in the surrounding fluid and the viscoelastic losses in both core and shell material, is analyzed in [20]. In a more related work, acoustic radiation impedances for axisymmetric modal oscillations of two neighboring spheres in a thermoviscous acoustic medium are calculated [21]. In the present paper, we undertake perhaps the simplest, detailed, acoustic analysis of eccentric multiple surfaces in a thermoviscous fluid. The principle objective is to investigate the effect of source eccentricity on radiation loading of (acoustic radiation from) a fluid-encapsulated sound projector immersed in a thermoviscous fluid. 


\section{Formulation}

\section{A. Basic Equations and Field Expansions}

There are a seemingly endless variety of models available now dealing with thermoviscous effects in acoustic wave propagation. The most exclusive model is based on a solution of the full set of basic governing equations, i.e., all terms in the linearized Navier Stokes equations are taken into account [22]. This inclusive treatment of thermoviscosity can greatly complicate the analysis because the fluid medium can then support shear and thermal as well as compressional modes, both of which must be accounted for in satisfying the boundary conditions at the interfaces. The problem considered here is that of computing the acoustic field of a spherical source vibrating with a time harmonic, axisymmetric, arbitrary surface velocity and eccentrically positioned within a thermoviscous fluid sphere that is embedded in another viscous thermally conducting (boundless) medium. The geometry and the coordinate systems used are depicted in Fig. 1. The classical Helmholtz decomposition expansion may be used advantageously to express fluid-particle velocity vector in the thermoviscous acoustic medium in terms of a compressional-wave scalar potential and a viscous-wave vector potential as [23]:

$$
u=-\nabla \varphi+\nabla \times \psi
$$

The governing equations for $\varphi, \psi$ and the excess temperature $T$ is then written as [22]:

$$
\begin{aligned}
-\omega^{2} \varphi+i \omega \frac{\alpha c^{2}}{\gamma} T & =\left(\frac{c^{2}}{\gamma}-i \omega \beta\right) \nabla^{2} \varphi \\
-i \omega T & =\frac{\kappa}{\rho C_{v}} \nabla^{2} T+\frac{\gamma-1}{\alpha} \nabla^{2} \varphi \\
-i \omega \psi & =v \nabla^{2} \psi,
\end{aligned}
$$

where $\kappa$ is the thermal conductivity, $C_{v}$ is the specific heat at constant volume, $\rho$ is the mass density, $\beta=4 v / 3+\mu_{b} / \rho$, $v=\mu / \rho$, is the kinematic viscosity, $\mu$ is shear (dynamic) viscosity, $\mu_{b}$ is the bulk (expansive) viscosity, $c$ is the adiabatic speed of sound, $\alpha$ is the coefficient of thermal expansion, $\gamma=C_{p} / C_{v}$ is the specific heat ratio, $C_{p}$ is the specific heat at constant pressure; and because the source is supposed to undergo time-harmonic surface oscillations with angular frequency $\omega$, harmonic time variations are assumed throughout with $e^{-i \omega t}$ dependence suppressed for simplicity.

The above governing equations may be algebraically manipulated to yield Helmholtz-type equations [22]:

$$
\begin{aligned}
\left(\nabla^{2}+k_{c}^{2}\right) \varphi_{c} & =0, \\
\left(\nabla^{2}+k_{t}^{2}\right) \varphi_{t} & =0, \\
\left(\nabla^{2}+k_{s}^{2}\right) \psi & =0,
\end{aligned}
$$

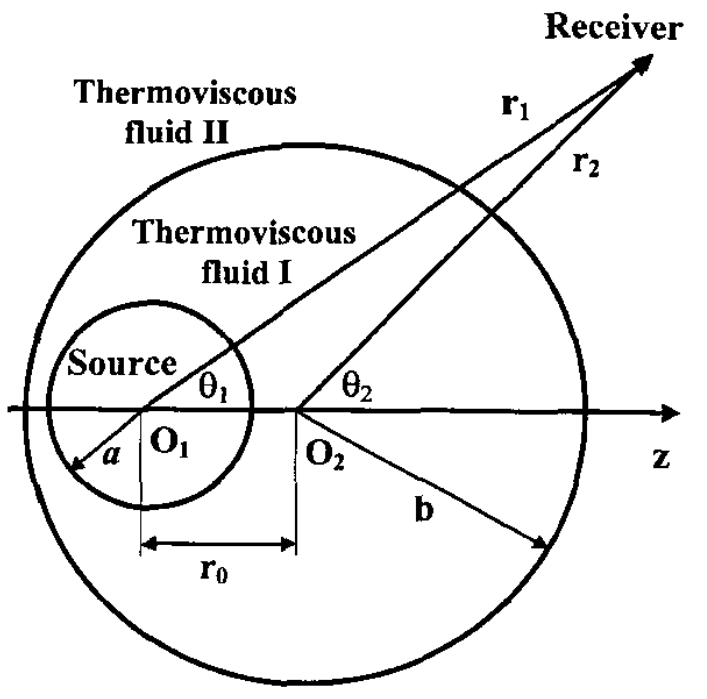

Fig. 1. Problem geometry.

where the subscripts $c, t$, and $s$ denote compressional, thermal, and shear, respectively. In addition, $\varphi=\varphi_{c}+\varphi_{t}$, and accurate approximations for $k_{c}, k_{t}$, and $k_{s}$ are given as [24]:

$$
\begin{aligned}
& k_{c}=\frac{\omega}{c}\left(1+i \frac{\omega \mu}{2 \rho c^{2}}\left(\frac{4}{3}+\frac{\mu_{b}}{\mu}+\frac{\gamma-1}{\operatorname{Pr}}\right)\right), \\
& k_{t}=(1+i) \sqrt{\omega / 2 \sigma} \\
& k_{s}=(1+i) \sqrt{\omega / 2 v}
\end{aligned}
$$

where $\operatorname{Pr}=\mu C_{p} / \kappa$ is Prandtl number and $\sigma=\kappa / \rho C_{p}$ is the thermal diffusivity.

For axisymmetric motion in spherical coordinates, $\psi=$ $(0 ., 0 ., \psi)$ and $\nabla$ is classical. Therefore, (1) yields for the radial and tangential velocities, and with $\varphi=\varphi_{c}+\varphi_{t}$, the first of (2) and the first two of (3) yield for the excess temperature [21]:

$$
\begin{aligned}
u_{r} & =\frac{\partial \varphi}{\partial r}+\frac{1}{r \sin \theta} \frac{\partial}{\partial \theta}(\psi \sin \theta), \\
u_{\theta} & =-\frac{1}{r} \frac{\partial \varphi}{\partial \theta}-\frac{1}{r} \frac{\partial}{\partial r}(r \psi), \\
T & =b_{c} \varphi_{c}+b_{t} \varphi_{t}
\end{aligned}
$$

where

$$
\begin{aligned}
& b_{c}=\frac{\gamma}{i \omega \alpha c^{2}}\left[\omega^{2}-k_{c}^{2}\left(\frac{c^{2}}{\gamma}-i \omega \beta\right)\right], \\
& b_{t}=\frac{\gamma}{i \omega \alpha c^{2}}\left[\omega^{2}-k_{t}^{2}\left(\frac{c^{2}}{\gamma}-i \omega \beta\right)\right] .
\end{aligned}
$$


In addition, the classical relations for radial and tangential stresses and heat flux may be used to yield the following potential-based expressions [25]:

$$
\begin{aligned}
\sigma_{r r} & =i \omega \rho \varphi \\
+ & 2 \mu\left[\nabla^{2} \varphi-\frac{\partial^{2} \varphi}{\partial r^{2}}+\frac{\partial}{\partial r}\left(\frac{1}{r \sin \theta} \frac{\partial}{\partial \theta}(\psi \sin \theta)\right)\right], \\
\sigma_{r \theta} & =\frac{\mu}{r}\left[-2 \frac{\partial^{2} \varphi}{\partial r \partial \theta}+\frac{2}{r} \frac{\partial \varphi}{\partial \theta}-\frac{\partial^{2}}{\partial r^{2}}(r \psi)\right. \\
& \left.+\frac{2}{r} \frac{\partial}{\partial r}(r \psi)+\frac{\partial}{\partial \theta}\left(\frac{1}{r \sin \theta} \frac{\partial}{\partial \theta}(\psi \sin \theta)\right)\right], \\
q= & -\kappa\left(b_{c} \frac{\partial \varphi_{c}}{\partial r}+b_{t} \frac{\partial \varphi_{t}}{\partial r}\right) .
\end{aligned}
$$

Mathematically, the dynamics of the problem may be expressed in terms of appropriate expansions. Accordingly, the velocity and temperature perturbations of the source surface, which are assumed to be symmetric about the $z$ axis but otherwise arbitrary, may be written as [21]:

$$
\begin{aligned}
& W\left(\theta_{1}, \omega\right)=\sum_{n=0}^{\infty} W_{n}(\omega) P_{n}\left(\cos \theta_{1}\right), \\
& V\left(\theta_{1}, \omega\right)=\sum_{n=1}^{\infty} V_{n}(\omega) P_{n}^{1}\left(\cos \theta_{1}\right), \\
& \bar{T}\left(\theta_{1}, \omega\right)=\sum_{n=0}^{\infty} \bar{T}_{n}(\omega) P_{n}\left(\cos \theta_{1}\right),
\end{aligned}
$$

where $P_{n}$ is Legendre polynomial, $P_{n}^{1}=-(d / d \theta) P_{n}$, and for a prescribed velocity and temperature distributions, Fourier coefficients $W_{n}(\omega), V_{n}(\omega)$, and $\tilde{T}_{n}(\omega)$ are known.

The interior fluid is denoted as region I, and the outer medium is denoted as region II (Fig. 1). In region $I$, the possibility of both incoming and outgoing (standing) waves exists, and in region II only outgoing waves are possible. Therefore, the solution of the Helmholtz equations for the acoustic velocity potentials in the fluid sphere may be represented by:

$$
\begin{aligned}
& \varphi_{c}^{I}\left(r_{1}, \theta_{1}, \omega\right)= \\
& \sum_{n=0}^{\infty}\left[a_{n} h_{n}^{(1)}\left(k_{c} r_{1}\right)+b_{n} h_{n}^{(2)}\left(k_{c} r_{1}\right)\right] P_{n}\left(\cos \theta_{1}\right), \\
& \varphi_{t}^{I}\left(r_{1}, \theta_{1}, \omega\right)= \\
& \sum_{n=0}^{\infty}\left[c_{n} h_{n}^{(1)}\left(k_{t} r_{1}\right)+d_{n} h_{n}^{(2)}\left(k_{t} r_{1}\right)\right] P_{n}\left(\cos \theta_{1}\right), \\
& \psi^{I}\left(r_{1}, \theta_{1}, \omega\right)= \\
& \sum_{n=1}^{\infty}\left[e_{n} h_{n}^{(1)}\left(k_{s} r_{1}\right)+f_{n} h_{n}^{(2)}\left(k_{s} r_{1}\right)\right] P_{n}^{1}\left(\cos \theta_{1}\right),
\end{aligned}
$$

where $h_{n}^{(1)}$ and $h_{n}^{(2)}$ are spherical Hankel functions of the first and second kind [26], respectively, and $a_{n}(\omega)$ through $f_{n}(\omega)$ are unknown modal coefficients. Similarly, keeping in mind the radiation condition, the outgoing solution can be expressed as:

$$
\begin{aligned}
& \varphi_{c}^{I I}\left(r_{2}, \theta_{2}, \omega\right)=\sum_{n=0}^{\infty} g_{n} h_{n}^{(1)}\left(k_{c}^{*} r_{2}\right) P_{n}\left(\cos \theta_{2}\right) \\
& \varphi_{t}^{I I}\left(r_{2}, \theta_{2}, \omega\right)=\sum_{n=0}^{\infty} s_{n} h_{n}^{(1)}\left(k_{t}^{*} r_{2}\right) P_{n}\left(\cos \theta_{2}\right) \\
& \psi^{I I}\left(r_{2}, \theta_{2}, \omega\right)=\sum_{n=1}^{\infty} x_{n} h_{n}^{(1)}\left(k_{s}^{*} r_{2}\right) P_{n}^{1}\left(\cos \theta_{2}\right)
\end{aligned}
$$

where the asterisks refer to the acoustic parameters in the (outer) fluid medium II.

\section{B. Addition Theorem and Boundary Conditions}

Many radiation and scattering problems involve waves of one characteristic shape (coordinate system) that are incident upon a boundary of some other shape (coordinate system). So it is difficult to satisfy the boundary conditions on that surface. To circumvent this difficulty in the current problem, one needs to express the spherical wave functions of the $\left(r_{1}, \theta_{1}, \vartheta_{1}\right)$ coordinate system in terms of spherical wave functions of the $\left(r_{2}, \theta_{2}, \vartheta_{2}\right)$ coordinate system through application of the classical form of translational addition theorem for bispherical coordinates [27]:

$$
\begin{aligned}
X_{n}\left(k r_{1}\right) P_{n}^{\varepsilon}\left(\cos \theta_{1}\right) & = \\
& \sum_{m=0}^{\infty} R_{n \varepsilon m 0} X_{m}\left(k r_{2}\right) P_{m}^{\epsilon}\left(\cos \theta_{2}\right),
\end{aligned}
$$

where $X_{n}$ is one of the spherical Bessel functions of order $n$, and

$$
\begin{aligned}
& R_{n \varepsilon m 0}= \\
& i^{m-n} \sum_{\lambda=|m-n|}^{m+n} i^{\lambda}(2 \lambda+1) b_{m}^{n \varepsilon \lambda 0} j_{\lambda}\left(k r_{0}\right) P_{\lambda}\left(\cos \theta_{0}\right)
\end{aligned}
$$

in which $\theta_{0}$ is the angle between the $O_{1} O_{2}$ line and the $z$ axis. Noting that there is a common $z$-axis in the current configuration (Fig. 1), we must set $\theta_{0}=0$ in (12). Furthermore, $b_{m}^{n \varepsilon \lambda 0}=(n \lambda 00 \mid m 0)(n \lambda \varepsilon 0 \mid m \varepsilon)$, in which Clebsch-Gordan coefficients are defined as shown in (13) (see next page), where the summation must be performed over the non-negative values of $z$ that make all factorials involving $z$ to be greater than or equal to zero.

Incorporation of the above addition theorem in (9) allows us to translate the wave components of the first coor- 


$$
\begin{aligned}
& \left(n_{1} n_{2} m_{1} m_{2} \mid n, m_{1}+m_{2}\right)=\left[\frac{2 n+1}{\left(n+n_{1}+n_{2}+1\right) !}\left(n+n_{1}-n_{2}\right) !\left(n_{1}+n_{2}-n\right) !\left(n_{2}+n-n_{1}\right) !\right. \\
& \left.\times\left(n_{1}-m_{1}\right) !\left(n_{1}+m_{1}\right) !\left(n_{2}-m_{2}\right) !\left(n_{2}+m_{2}\right) !\left(n+m_{1}+m_{2}\right) !\left(n-m_{1}-m_{2}\right) !\right]^{\frac{1}{2}} \\
& \times \sum_{z}\left\{( - 1 ) ^ { z } \left[z !\left(n_{1}+n_{2}-n-z\right) !\left(z+n-n_{1}-m_{2}\right) !\left(n_{2}+m_{2}-z\right) !\right.\right. \\
& \left.\left.\quad \times\left(z+n-n_{2}+m_{1}\right) !\left(n_{1}-m_{1}-z\right) !\right]^{-1}\right\}
\end{aligned}
$$

dinate system in terms of spherical wave functions of the second coordinate system, i.e.:

$$
\begin{aligned}
& \varphi_{c}^{I}\left(r_{2}, \theta_{2}, \omega\right)= \\
& \sum_{n=0}^{\infty}\left[A_{n} h_{n}^{(1)}\left(k_{c} r_{2}\right)+B_{n} h_{n}^{(2)}\left(k_{c} r_{2}\right)\right] P_{n}\left(\cos \theta_{2}\right) \\
& \varphi_{t}^{I}\left(r_{2}, \theta_{2}, \omega\right)= \\
& \sum_{n=0}^{\infty}\left[C_{n} h_{n}^{(1)}\left(k_{t} r_{2}\right)+D_{n} h_{n}^{(2)}\left(k_{t} r_{2}\right)\right] P_{n}\left(\cos \theta_{2}\right),(14) \\
& \psi^{I}\left(r_{2}, \theta_{2}, \omega\right)= \\
& \sum_{n=1}^{\infty}\left[E_{n} h_{n}^{(1)}\left(k_{s} r_{2}\right)+F_{n} h_{n}^{(2)}\left(k_{s} r_{2}\right)\right] P_{n}^{1}\left(\cos \theta_{2}\right)
\end{aligned}
$$

where

$$
\begin{aligned}
& A_{n}=\sum_{m=0}^{\infty} R_{m 0 n 0} a_{m}, B_{n}=\sum_{m=0}^{\infty} R_{m 0 n 0} b_{m} \\
& C_{n}=\sum_{m=0}^{\infty} R_{m 0 n 0} c_{m}, \quad D_{n}=\sum_{m=0}^{\infty} R_{m 0 n 0} d_{m} \\
& E_{n}=\sum_{m=1}^{\infty} R_{m 1 n 0} e_{m}, F_{n}=\sum_{m=1}^{\infty} R_{m 1 n 0} f_{m}
\end{aligned}
$$

The unknown modal coefficients $a_{n}(\omega)$ through $x_{n}(\omega)$ must be determined by imposing the suitable boundary conditions. Correspondingly, the continuity of radial and tangential velocity components, and the perturbation in temperature at the surface of the source require that:

$$
\begin{aligned}
& W\left(\theta_{1}, \omega\right)=u_{r}^{I}\left(r_{1}=a, \theta_{1}, \omega\right), \\
& V\left(\theta_{1}, \omega\right)=u_{\theta}^{I}\left(r_{1}=a, \theta_{1}, \omega\right), \\
& \tilde{T}\left(\theta_{1}, \omega\right)=T\left(r_{1}=a, \theta_{1}, \omega\right)=0,
\end{aligned}
$$

where the perturbation in temperature at the surface of the source is assumed to remain zero, because in many applications the thermal conductivity of the radiator (scatterer) greatly exceeds that of the surrounding acoustic medium. Similarly, the continuity of the normal and tangential velocity components, temperature, heat flux, and normal and tangential stress components at the fluid interface demands that:

$$
\begin{aligned}
& u_{r}^{I I}\left(r_{2}=b, \theta_{2}, \omega\right)=u_{r}^{I}\left(r_{2}=b, \theta_{2}, \omega\right), \\
& \sigma_{r r}^{I I}\left(r_{2}=b, \theta_{2}, \omega\right)=\sigma_{r r}^{I}\left(r_{2}=b, \theta_{2}, \omega\right), \\
& u_{\theta}^{I I}\left(r_{2}=b, \theta_{2}, \omega\right)=u_{\theta}^{I}\left(r_{2}=b, \theta_{2}, \omega\right), \\
& \sigma_{r \theta}^{I I}\left(r_{2}=b, \theta_{2}, \omega\right)=\sigma_{r \theta}^{I}\left(r_{2}=b, \theta_{2}, \omega\right), \\
& T^{I I}\left(r_{2}=b, \theta_{2}, \omega\right)=T^{I}\left(r_{2}=b, \theta_{2}, \omega\right), \\
& q^{I I}\left(r_{2}=b, \theta_{2}, \omega\right)=q^{I}\left(r_{2}=b, \theta_{2}, \omega\right) .
\end{aligned}
$$

Now the unknown modal coefficients can be determined by using the translational addition theorem in the stated boundary conditions. Therefore, using expansions (8), (9), (10), and (14) in the boundary conditions (16) and (17), we obtain (18) (see next page).

\section{Modal Acoustic Radiation Impedance}

The fluctuating fluid stresses on the surface of the vibrating source constitute its radiation loading. The radiation loading on the spherical surface excited in vibrational modes of various order (i.e., monopole, dipole, quadrupole, and other multipole-like radiators), as described through an acoustic radiation impedance matrix, may be determined in a few simple steps as follows [21]. First, from (9) and the first two of (7), the modal components of the normal and tangential stresses on the surface of the source are expressed as shown in (19) (see page 1449) which can be put in the truncated matrix form:

$$
\boldsymbol{\sigma}=\mathbf{S}\left(r_{0}, \omega\right) \mathbf{c}
$$

where $\mathbf{S}$ is a $(2 N+1) \times(9 N+6)$ rectangular matrix that contains the coefficients multiplying the modal parameters $a_{n}$ through $f_{n}$ in the modal stress relations (19), and:

$\sigma=\left[\sigma_{r r}^{0}, \sigma_{r r}^{1}, \ldots, \sigma_{r r}^{N} ; \sigma_{r \theta}^{1}, \sigma_{r \theta}^{2}, \ldots, \sigma_{r \theta}^{N}\right]$,

$\mathbf{c}=\left[a_{0}, a_{1}, \ldots, a_{N} ; b_{0}, b_{1}, \ldots, b_{N} ; \ldots ; x_{0}, x_{1}, \ldots, x_{N}\right]^{T}$

Next, the truncated systems of (18a) through (18i) are advantageously put in the matrix form:

$$
u=R\left(r_{0}, \omega\right) c
$$




$$
\begin{aligned}
& -k_{c}\left[a_{n} h_{n}^{(1)^{\prime}}\left(k_{c} a\right)+b_{n} h_{n}^{(2)^{\prime}}\left(k_{c} a\right)\right]-k_{t}\left[c_{n} h_{n}^{(1)^{\prime}}\left(k_{t} a\right)+d_{n} h_{n}^{(2)^{\prime}}\left(k_{t} a\right)\right]+\frac{n(n+1)}{a}\left[e_{n} h_{n}^{(1)}\left(k_{s} a\right)+f_{n} h_{n}^{(2)}\left(k_{s} a\right)\right]=W_{n} \\
& \frac{1}{a}\left[a_{n} h_{n}^{(1)}\left(k_{c} a\right)+b_{n} h_{n}^{(2)}\left(k_{c} a\right)\right]+\frac{1}{a}\left[c_{n} h_{n}^{(1)}\left(k_{t} a\right)+d_{n} h_{n}^{(2)}\left(k_{t} a\right)\right] \\
& -\left\{\left[\frac{h_{n}^{(1)}\left(k_{s} a\right)}{a}+k_{s} h_{n}^{(1)^{\prime}}\left(k_{s} a\right)\right] e_{n}+\left[\frac{h_{n}^{(2)}\left(k_{s} a\right)}{a}+k_{s} h_{n}^{(2)^{\prime}}\left(k_{s} a\right)\right] f_{n}\right\}=V_{n}, \\
& b_{c}\left[a_{n} h_{n}^{(1)}\left(k_{c} a\right)+b_{n} h_{n}^{(2)}\left(k_{c} a\right)\right]+b_{t}\left[c_{n} h_{n}^{(1)}\left(k_{t} a\right)+d_{n} h_{n}^{(2)}\left(k_{t} a\right)\right]=0 \\
& -k_{c}\left[A_{n} h_{n}^{(1)^{\prime}}\left(k_{c} b\right)+B_{n} h_{n}^{(2)^{\prime}}\left(k_{c} b\right)\right]-k_{t}\left[C_{n} h_{n}^{(1)^{\prime}}\left(k_{t} b\right)+D_{n} h_{n}^{(2)^{\prime}}\left(k_{t} b\right)\right] \\
& +\frac{n(n+1)}{b}\left[E_{n} h_{n}^{(1)}\left(k_{s} b\right)+F_{n} h_{n}^{(2)}\left(k_{s} b\right)\right]+k_{c}^{*} g_{n} h_{n}^{(1)^{\prime}}\left(k_{c}^{*} b\right)+k_{t}^{*} s_{n} h_{n}^{(1)^{\prime}}\left(k_{t}^{*} b\right)-\frac{n(n+1)}{b} x_{n} h_{n}^{(1)}\left(k_{s}^{*} b\right)=0 \\
& \frac{1}{b}\left[A_{n} h_{n}^{(1)}\left(k_{c} b\right)+B_{n} h_{n}^{(2)}\left(k_{c} b\right)\right]+\frac{1}{b}\left[C_{n} h_{n}^{(1)}\left(k_{t} b\right)+D_{n} h_{n}^{(2)}\left(k_{t} b\right)\right]-\left[\frac{1}{b} h_{n}^{(1)}\left(k_{s} b\right)+k_{s} h_{n}^{(1)^{\prime}}\left(k_{s} b\right)\right] E_{n} \\
& -\left[\frac{1}{b} h_{n}^{(2)}\left(k_{s} b\right)+k_{s} h_{n}^{(2)^{\prime}}\left(k_{s} b\right)\right] F_{n}-\frac{1}{b} g_{n} h_{n}^{(1)}\left(k_{c}^{*} b\right)-\frac{1}{b} s_{n} h_{n}^{(1)}\left(k_{t}^{*} b\right)+\left[\frac{1}{b} h_{n}^{(1)}\left(k_{s}^{*} b\right)+k_{s}^{*} h_{n}^{(1)^{\prime}}\left(k_{s}^{*} b\right)\right] x_{n}=0, \\
& b_{c}\left[A_{n} h_{n}^{(1)}\left(k_{c} b\right)+B_{n} h_{n}^{(2)}\left(k_{c} b\right)\right]+b_{t}\left[C_{n} h_{n}^{(1)}\left(k_{t} b\right)+D_{n} h_{n}^{(2)}\left(k_{t} b\right)\right]-b_{c}^{*} g_{n} h_{n}^{(1)}\left(k_{c}^{*} b\right)-b_{t}^{*} s_{n} h_{n}^{(1)}\left(k_{t}^{*} b\right)=0, \\
& b_{c} \kappa k_{c}\left[A_{n} h_{n}^{(1)^{\prime}}\left(k_{c} b\right)+B_{n} h_{n}^{(2)^{\prime}}\left(k_{c} b\right)\right]+b_{t} \kappa k_{t}\left[C_{n} h_{n}^{(1)^{\prime}}\left(k_{t} b\right)+D_{n} h_{n}^{(2)^{\prime}}\left(k_{t} b\right)\right] \\
& -b_{c}^{*} \kappa^{*} k_{c}^{*} g_{n} h_{n}^{(1)^{\prime}}\left(k_{c}^{*} b\right)-b_{t}^{*} \kappa^{*} k_{t}^{*} s_{n} h_{n}^{(1)^{\prime}}\left(k_{t}^{*} b\right)=0, \\
& {\left[i \omega \rho h_{n}^{(1)}\left(k_{c} b\right)-2 \mu k_{c}^{2} h_{n}^{(1)}\left(k_{c} b\right)-2 \mu k_{c}^{2} h_{n}^{(1)^{\prime \prime}}\left(k_{c} b\right)\right] A_{n}+\left[i \omega \rho h_{n}^{(2)}\left(k_{c} b\right)-2 \mu k_{c}^{2} h_{n}^{(2)}\left(k_{c} b\right)-2 \mu k_{c}^{2} h_{n}^{(2)^{\prime \prime}}\left(k_{c} b\right)\right] B_{n}} \\
& +\left[i \omega \rho h_{n}^{(1)}\left(k_{t} b\right)-2 \mu k_{t}^{2} h_{n}^{(1)}\left(k_{t} b\right)-2 \mu k_{t}^{2} h_{n}^{(1)^{\prime \prime}}\left(k_{t} b\right)\right] C_{n}+\left[i \omega \rho h_{n}^{(2)}\left(k_{t} b\right)-2 \mu k_{t}^{2} h_{n}^{(2)}\left(k_{t} b\right)-2 \mu k_{t}^{2} h_{n}^{(2)^{\prime \prime}}\left(k_{t} b\right)\right] D_{n} \\
& +\left\{\frac{2 \mu n(n+1)}{b^{2}}\left[b k_{s} h_{n}^{(1)^{\prime}}\left(k_{s} b\right)-h_{n}^{(1)}\left(k_{s} b\right)\right]\right\} E_{n}+\left\{\frac{2 \mu n(n+1)}{b^{2}}\left[b k_{s} h_{n}^{(2)^{\prime}}\left(k_{s} b\right)-h_{n}^{(2)}\left(k_{s} b\right)\right]\right\} F_{n} \\
& -\left[i \omega \rho^{*} h_{n}^{(1)}\left(k_{c}^{*} b\right)-2 \mu^{*} k_{c}^{*^{2}} h_{n}^{(1)}\left(k_{c}^{*} b\right)-2 \mu^{*} k_{c}^{*^{2}} h_{n}^{(1)^{\prime \prime}}\left(k_{c}^{*} b\right)\right] g_{n}-\left[i \omega \rho^{*} h_{n}^{(1)}\left(k_{t}^{*} b\right)-2 \mu^{*} k_{t}^{*^{2}} h_{n}^{(1)}\left(k_{t}^{*} b\right)-2 \mu^{*} k_{t}^{*^{2}} h_{n}^{(1)^{\prime \prime}}\left(k_{t}^{*} b\right)\right] s_{n} \\
& -\left\{\frac{2 \mu^{*} n(n+1)}{b^{2}}\left[b k_{s}^{*} h_{n}^{(1)^{\prime}}\left(k_{s}^{*} b\right)-h_{n}^{(1)}\left(k_{s}^{*} b\right)\right]\right\} x_{n}=0 \\
& \frac{2 \mu}{b^{2}}\left[b k_{c} h_{n}^{(1)^{\prime}}\left(k_{c} b\right)-h_{n}^{(1)}\left(k_{c} b\right)\right] A_{n}+\frac{2 \mu}{b^{2}}\left[b k_{c} h_{n}^{(2)^{\prime}}\left(k_{c} b\right)-h_{n}^{(2)}\left(k_{c} b\right)\right] B_{n}+\frac{2 \mu}{b^{2}}\left[b k_{t} h_{n}^{(1)^{\prime}}\left(k_{t} b\right)-h_{n}^{(1)}\left(k_{t} b\right)\right] C_{n} \\
& +\frac{2 \mu}{b^{2}}\left[b k_{t} h_{n}^{(2)^{\prime}}\left(k_{t} b\right)-h_{n}^{(2)}\left(k_{t} b\right)\right] D_{n}+\mu\left[\frac{2-n(n+1)}{b^{2}} h_{n}^{(1)}\left(k_{s} b\right)-k_{s}^{2} h_{n}^{(1)^{\prime \prime}}\left(k_{s} b\right)\right] E_{n} \\
& +\mu\left[\frac{2-n(n+1)}{b^{2}} h_{n}^{(2)}\left(k_{s} b\right)-k_{s}^{2} h_{n}^{(2)^{\prime \prime}}\left(k_{s} b\right)\right] F_{n}-\frac{2 \mu^{*}}{b^{2}}\left[b k_{c}^{*} h_{n}^{(1)^{\prime}}\left(k_{c}^{*} b\right)-h_{n}^{(1)}\left(k_{c}^{*} b\right)\right] g_{n} \\
& -\frac{2 \mu^{*}}{b^{2}}\left[b k_{t}^{*} h_{n}^{(1)^{\prime}}\left(k_{c}^{*} b\right)-h_{n}^{(1)}\left(k_{c}^{*} b\right)\right] s_{n}-\mu^{*}\left[\frac{2-n(n+1)}{b^{2}} h_{n}^{(1)}\left(k_{s}^{*} b\right)-k_{s}^{*^{2}} h_{n}^{(1)^{\prime \prime}}\left(k_{s}^{*} b\right)\right] x_{n}=0 .
\end{aligned}
$$

in which $R$ is a $(9 N+6) \times(9 N+6)$ square matrix whose elements are the coefficients multiplying the modal parameters $a_{n}$ through $x_{n}$ in the boundary conditions (18), and $u=\left[W_{0}, W_{1}, \ldots, W_{N} ; V_{1}, \ldots, V_{N} ; 0 ., 0, \ldots, 0 .\right]^{T}$.

Incorporating (22) in (20), the column vector containing the modal surface stresses can be expressed conveniently in terms of modal surface velocities as:

$$
\sigma=Z\left(r_{0}, \omega\right) u
$$

where the modal acoustic impedance matrix $Z\left(r_{0}, \omega\right)$ is given by:

$$
Z\left(r_{0}, \omega\right)=S\left(r_{0}, \omega\right) R^{-1}\left(r_{0}, \omega\right)
$$

where each element of the above fully populated $(9 \mathrm{~N}-$ 3) $\times(9 N-3)$ impedance matrix may beneficially be put in terms of a resistive and a reactive component as [28]:

$$
z_{n m}\left(r_{0}, \omega\right)=r_{n m}\left(r_{0}, \omega\right)-i \omega m_{n m}\left(r_{0}, \omega\right) .
$$




$$
\begin{array}{r}
\sigma_{r r}^{n}=\left[i \omega \rho h_{n}^{(1)}\left(k_{c} a\right)-2 \mu k_{c}^{2} h_{n}^{(1)}\left(k_{c} a\right)-2 \mu k_{c}^{2} h_{n}^{(1)^{\prime \prime}}\left(k_{c} a\right)\right] a_{n}+\left[i \omega \rho h_{n}^{(2)}\left(k_{c} a\right)-2 \mu k_{c}^{2} h_{n}^{(2)}\left(k_{c} a\right)-2 \mu k_{c}^{2} h_{n}^{(2)^{\prime \prime}}\left(k_{c} a\right)\right] b_{n} \\
+\left[i \omega \rho h_{n}^{(1)}\left(k_{t} a\right)-2 \mu k_{t}^{2} h_{n}^{(1)}\left(k_{t} a\right)-2 \mu k_{t}^{2} h_{n}^{(1)^{\prime \prime}}\left(k_{t} a\right)\right] c_{n}+\left[i \omega \rho h_{n}^{(2)}\left(k_{t} a\right)-2 \mu k_{t}^{2} h_{n}^{(2)}\left(k_{t} a\right)-2 \mu k_{t}^{2} h_{n}^{(2)^{\prime \prime}}\left(k_{t} a\right)\right] d_{n} \\
+\left\{\frac{2 \mu n(n+1)}{a^{2}}\left[a k_{s} h_{n}^{(1)^{\prime}}\left(k_{s} a\right)-h_{n}^{(1)}\left(k_{s} a\right)\right]\right\} e_{n}+\left\{\frac{2 \mu n(n+1)}{a^{2}}\left[a k_{s} h_{n}^{(2)^{\prime}}\left(k_{s} a\right)-h_{n}^{(2)}\left(k_{s} a\right)\right]\right\} f_{n} \\
\sigma_{r \theta}^{n}=\frac{2 \mu}{a^{2}}\left[a k_{c} h_{n}^{(1)^{\prime}}\left(k_{c} a\right)-h_{n}^{(1)}\left(k_{c} a\right)\right] a_{n}+\frac{2 \mu}{a^{2}}\left[a k_{c} h_{n}^{(2)^{\prime}}\left(k_{c} a\right)-h_{n}^{(2)}\left(k_{c} a\right)\right] b_{n}+\frac{2 \mu}{a^{2}}\left[a k_{t} h_{n}^{(1)^{\prime}}\left(k_{t} a\right)-h_{n}^{(1)}\left(k_{t} a\right)\right] c_{n} \\
+\frac{2 \mu}{a^{2}}\left[a k_{c} h_{n}^{(2)^{\prime}}\left(k_{t} a\right)-h_{n}^{(2)}\left(k_{t} a\right)\right] d_{n}+\mu\left[\frac{2-n(n+1)}{a^{2}} h_{n}^{(1)}\left(k_{s} a\right)-k_{s}^{2} h_{n}^{(1)^{\prime \prime}}\left(k_{s} a\right)\right] e_{n} \\
+\mu\left[\frac{2-n(n+1)}{a^{2}} h_{n}^{(2)}\left(k_{s} a\right)-k_{s}^{2} h_{n}^{(2)^{\prime \prime}}\left(k_{s} a\right)\right] f_{n}
\end{array}
$$

TABLE I

Parameter Values Used in Calculations.

\begin{tabular}{lcc}
\hline Parameter & Water & FC-75 \\
\hline $\mathrm{c}(\mathrm{cm} / \mathrm{s})$ & 149700 & 61377 \\
$\rho\left(\mathrm{g} / \mathrm{cm}^{3}\right)$ & 0.997 & 1.73 \\
$\mu\left(\mathrm{g} / \mathrm{cm} \mathrm{s}^{3}\right)$ & 0.00894 & 0.0079 \\
$\mu_{b}(\mathrm{~g} / \mathrm{cm} \mathrm{s})$ & 0.0250 & 0.0079 \\
$C_{p}\left(\mathrm{erg} / \mathrm{g}^{\circ} \mathrm{K}\right)$ & $4.179 \times 10^{7}$ & $1.044 \times 10^{7}$ \\
$C_{v}\left(\mathrm{erg} / \mathrm{g}^{\circ} \mathrm{K}\right)$ & $4.138 \times 10^{7}$ & $1.038 \times 10^{7}$ \\
$\alpha\left({ }^{\circ} \mathrm{K}-1\right)$ & 0.000257 & 0.001799 \\
$\kappa\left(\mathrm{dyn} / \mathrm{s}^{\circ} \mathrm{K}\right)$ & 59500 & 6398 \\
\hline
\end{tabular}

\section{NUMERICAL RESULTS}

In order to illustrate the nature and general behavior of the solution, we consider a numerical example in this section. Realizing the large number of parameters and the very intense computations involved here, no attempt is made to exhaustively evaluate the effect of varying each of them. The intent of the collection of data presented here is merely to illustrate the kinds of results to be expected from some representative and physically realistic choices of values for these parameters. From these data some trends are noted and general conclusions are made about the relative importance of certain parameters. Keeping in view the availability of numerical data, we shall confine our attention to a particular model. The surrounding ambient fluid is assumed to be water at atmospheric pressure and 300 degrees Kelvin. The interior fluid is selected as "Fluorinert" chemical FC-75 (3M, St. Paul, MN). The source is assumed to be of dimension $a=0.1 b=0.01 \mathrm{~cm}$, which can vibrate in pulsating $(n=0)$ or oscillating $(n=1)$ modes. The numerical values for the input parameters, which are used in the calculations, are summarized in Table I [29], [30]. Subsequently, a MAPLE code was constructed for treating boundary conditions, to compute the modal impedance matrix, $Z=S R^{-1}$, and to calculate the far-field radiated pressure as a function of source eccentricity at selected nondimensional frequencies, $k b=\omega b / c$. Derivatives of spherical Bessel functions were computed by using (10.1.19) and (10.1.22) in [26]. Noting the inca- pability of symbolic matrix inversion process in MAPLE linear algebra library to invert relatively large matrices, an independent MAPLE code for numerical matrix inversion using Gauss-Jordan (GAUSSJ) elimination with full pivoting based on subroutine GAUSSJ in section 2.1 of [31] was constructed. In addition, because the inaccurate MAPLE computations for Legendre functions of nonzero order, a MAPLE code based on FUNCTION PLGNDR in section 6.6 of [31] was worked out. The computations were simultaneously performed on about 30 Pentium III personal computers with a truncation constant of $N=40$ to assure convergence in the high-frequency range and in case of high-source eccentricity. Consequently, over $20000 \mathrm{com}$ puter hours had to be spent to obtain the final numerical results. Moreover, to examine thermoviscous effects more systematically, an additional MAPLE code was developed for solving the corresponding ideal (inviscid) fluid problem. It was successfully tested by accurately reproducing all the numerical results presented in [12].

Figs. 2 through 5 present the effect of source eccentricity on the inertial and the resistive components of the modal acoustic impedance for the source vibrating in $\mathrm{n}=0$ and $\mathrm{n}=1$ modes at selected nondimensional frequencies $\mathrm{kb}=10,20$. Comparison of these figures leads to the following important observations. First, we note greater variations in modal resistance and inertia values at $\mathrm{kb}=20$ relative to the lower $\mathrm{kb}=10$ frequency. Second, we observe fairly higher (lower) modal resistance (reactance) overall magnitudes at $\mathrm{kb}=20$ for almost all source eccentricities and for both modes of source oscillations as compared to the $\mathrm{kb}=10$ case. Moreover, there appears to be a relatively high peak (primary focal point) in the modal resistance curve at $\mathrm{kb}=20$ near the source eccentricity of $\mathrm{r}_{0} / \mathrm{b}=0.7$ for dipole (monopole) oscillations in the thermoviscous (ideal) fluid. This simply suggests that a comparatively higher sound power radiation at the noted source eccentricity is to be expected. Ultimately, we make the important observation that the full inclusion of fluid thermoviscosity has lead to a general increase in the resistive and reactive components of the modal impedance values for most source eccentricities and for both modes of source oscillations, especially at $\mathrm{kb}=20$. 


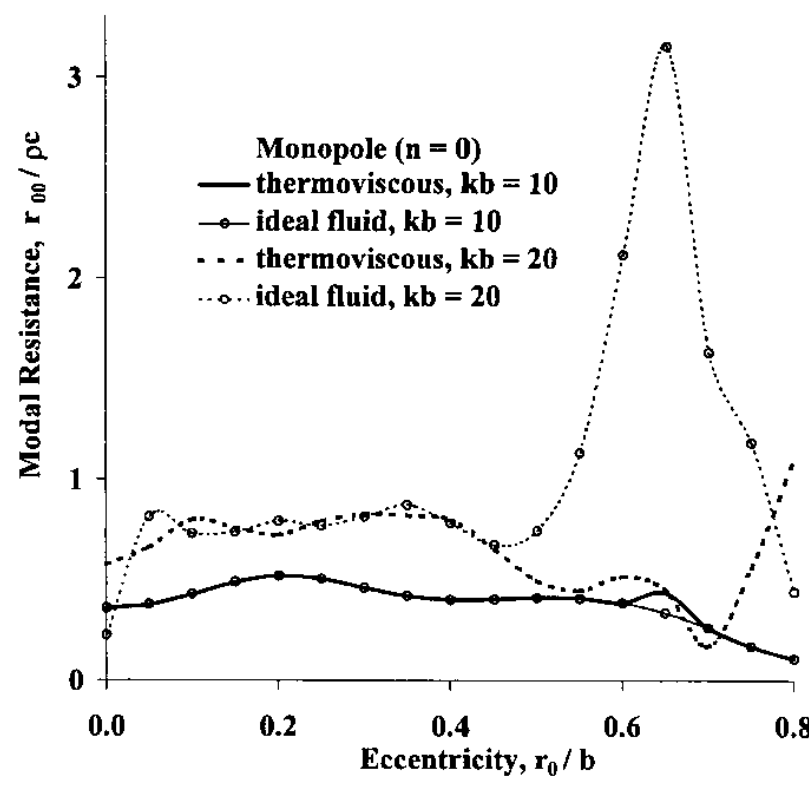

Fig. 2. Effect of source eccentricity on the resistive component of the modal acoustic radiation impedance load upon the pulsating source at selected nondimensional frequencies.

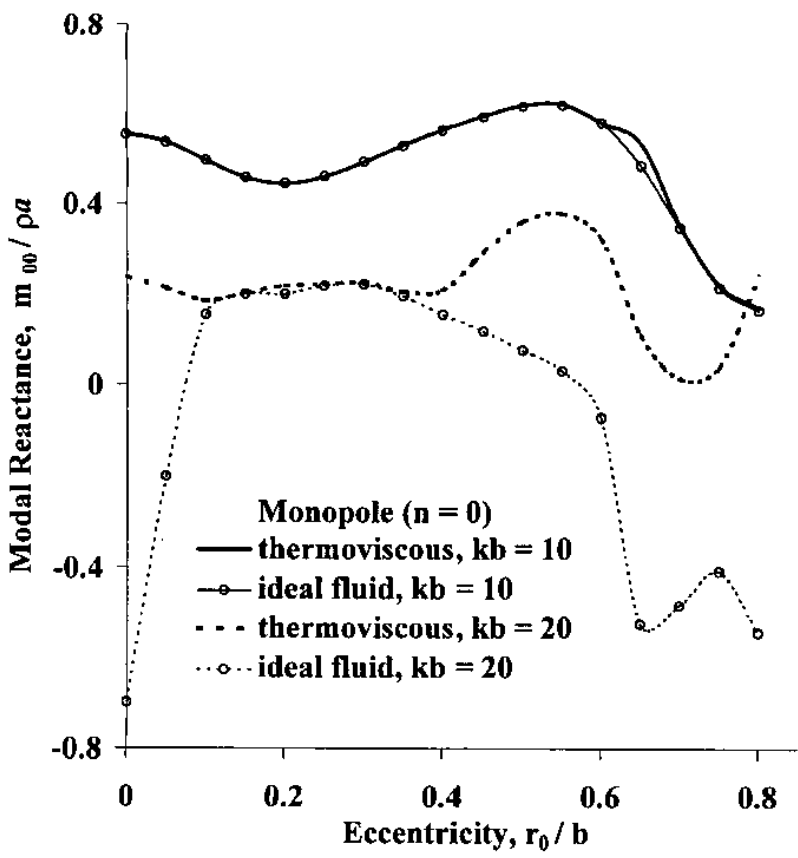

Fig. 3. Effect of source eccentricity on the reactive component of the modal acoustic radiation impedance load upon the pulsating source at selected nondimensional frequencies.

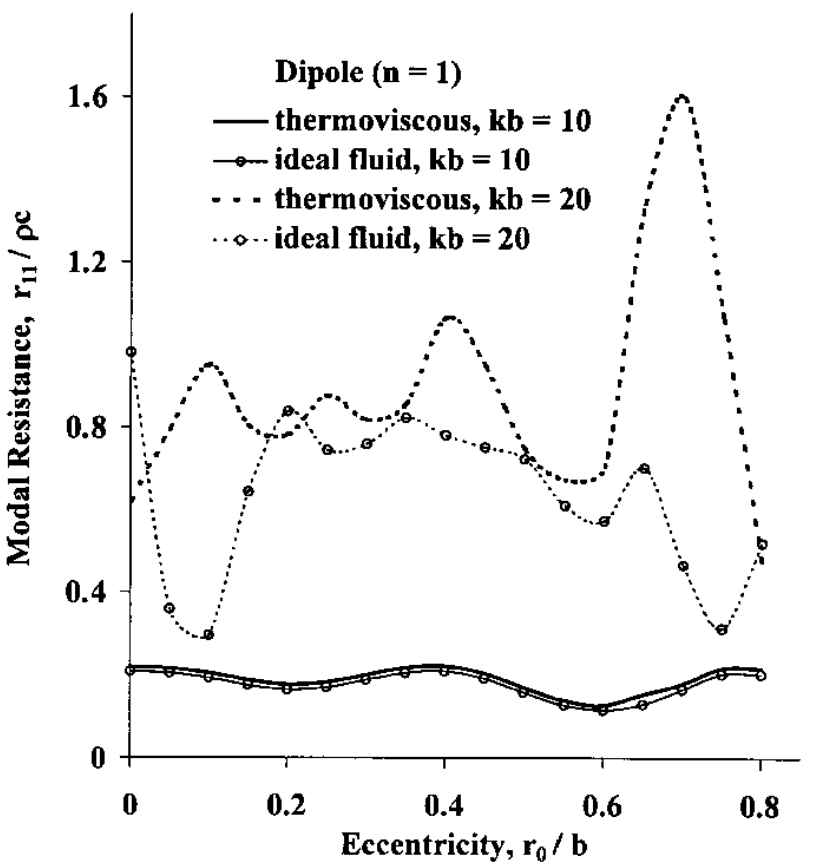

Fig. 4. Effect of source eccentricity on the resistive component of the modal acoustic radiation impedance load upon the oscillating source at selected nondimensional frequencies.

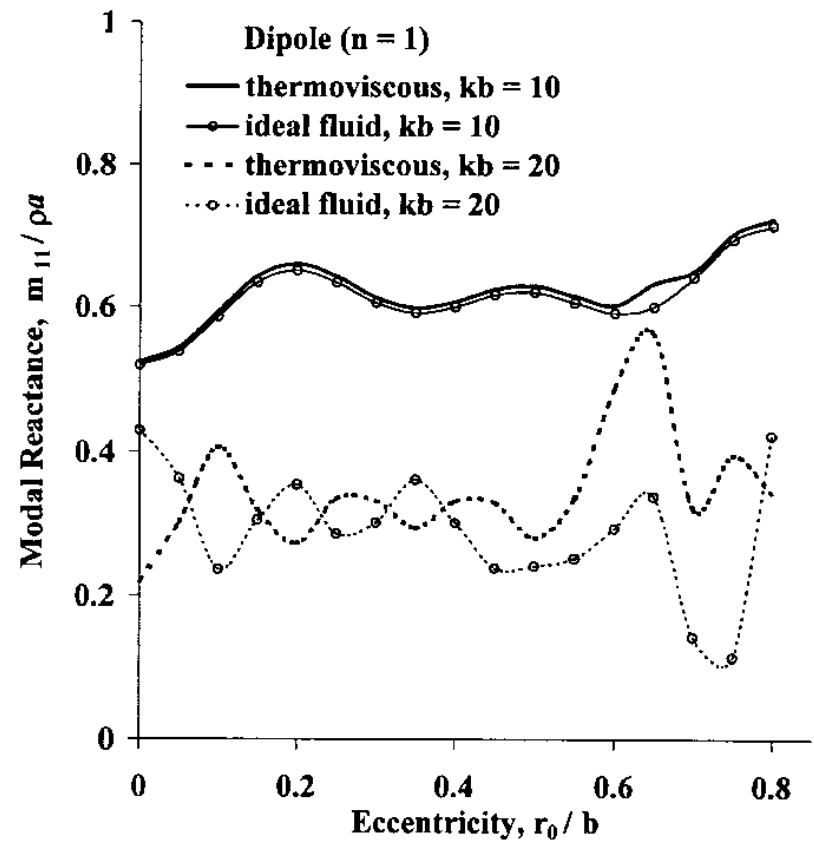

Fig. 5. Effect of source eccentricity on the reactive component of the modal acoustic radiation impedance load upon the oscillating source at selected nondimensional frequencies. 


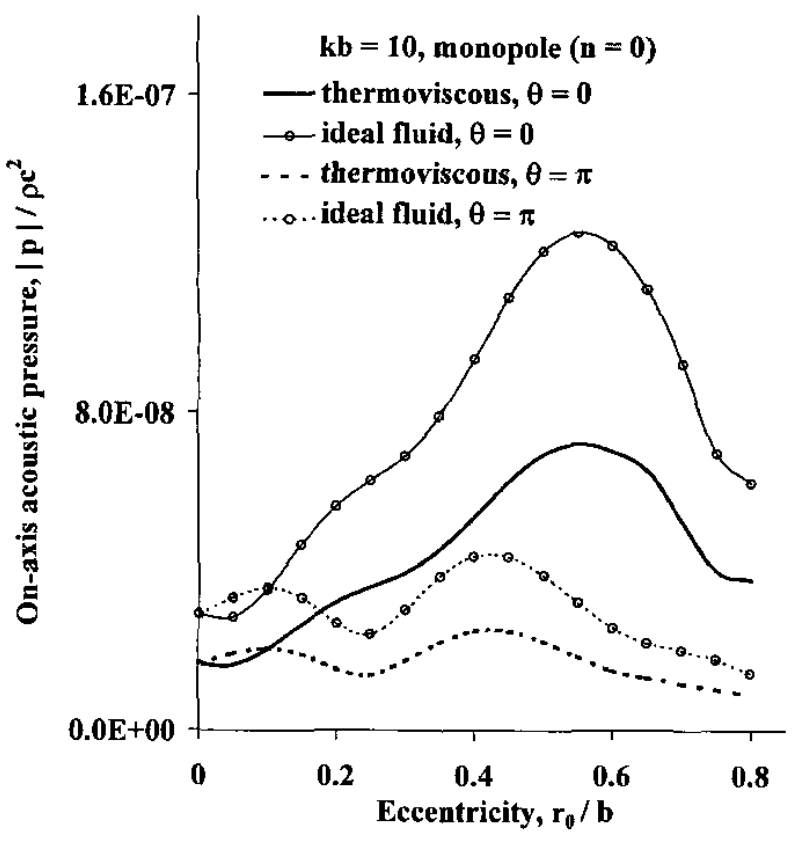

Fig. 6. Influence of source eccentricity on the on-axis radiated farfield acoustic pressures for the source vibrating in $n=0$ mode at $\mathrm{kb}=10$.

Figs. 6 through 9 display the influence of source eccentricity on the on-axis radiated far-field acoustic pressures [12] (i.e., at $\theta=0, \pi$ ), in which the source vibrating in $\mathrm{n}=0$ and $\mathrm{n}=1$ modes at the selected dimensionless wave numbers. The far-field value of the radial coordinate in each case is simply chosen by making several computer runs while seeking for the convergence of the radiated pressure. The choice of $r_{\infty}=20 \mathrm{~b}(=2 \mathrm{~cm})$ is found to be adequate for all cases. It is very informative to study the change in the on-axis output of the radiated waves as the source eccentricity is varied. We first note a distinctively lower on-axis pressure magnitude in the thermoviscous fluid as compared to the ideal fluid case in all figures. Next, we notice that the monopole on-axis pressure curves are less oscillatory with a larger overall magnitude and generally one main focal point in contrast to the dipole pressures with normally two comparable maxima (i.e., a primary and a somewhat smaller secondary focal point). We further see that, unlike the $k b=20$ case, the on-axis thermoviscous pressure curves at $\mathrm{kb}=10$ are almost identical in shape to the corresponding ideal fluid results (i.e., they share the same focal points).

Figs. 10 through 13 exhibit the angular distribution of the radiated far-field pressure for the source vibrating in $\mathrm{n}=0$ and $\mathrm{n}=1$ modes at selected source eccentricities and nondimensional wave numbers. The eccentricity values marked by "** correspond to the primary focal points of the on-axis radiated far-field acoustic pressures at $\theta=0$ as observed in Figs. 6 through 9. Here, we first note that the sharpness (narrowness) of the main lobe dramatically increases when the source is placed at a primary

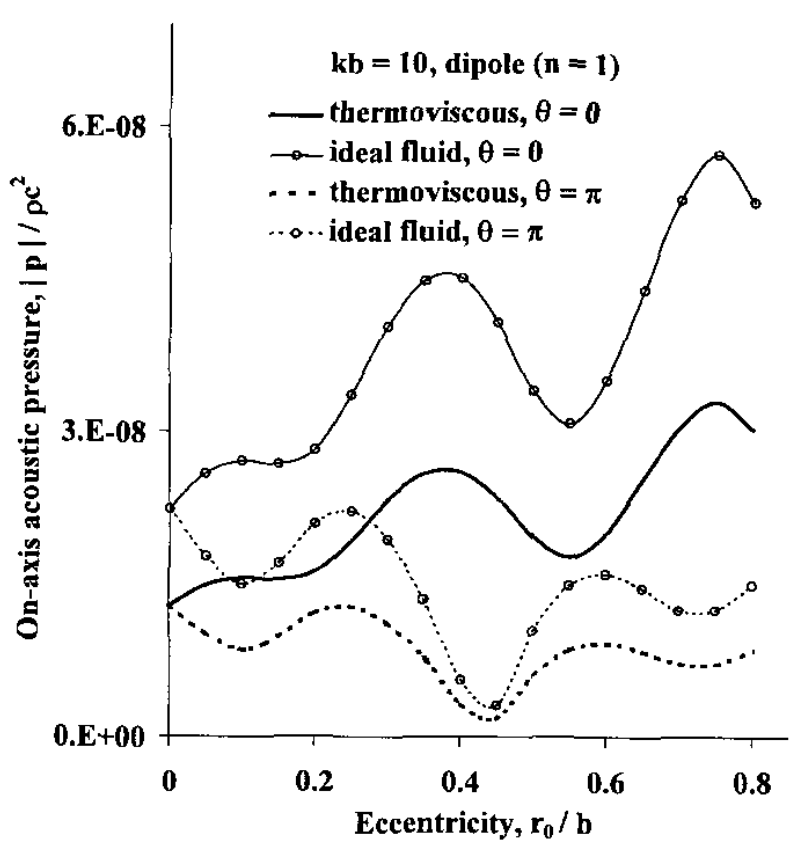

Fig. 7. Influence of source eccentricity on the on-axis radiated farfield acoustic pressures for the source vibrating in $n=1$ mode at $\mathrm{kb}=10$.

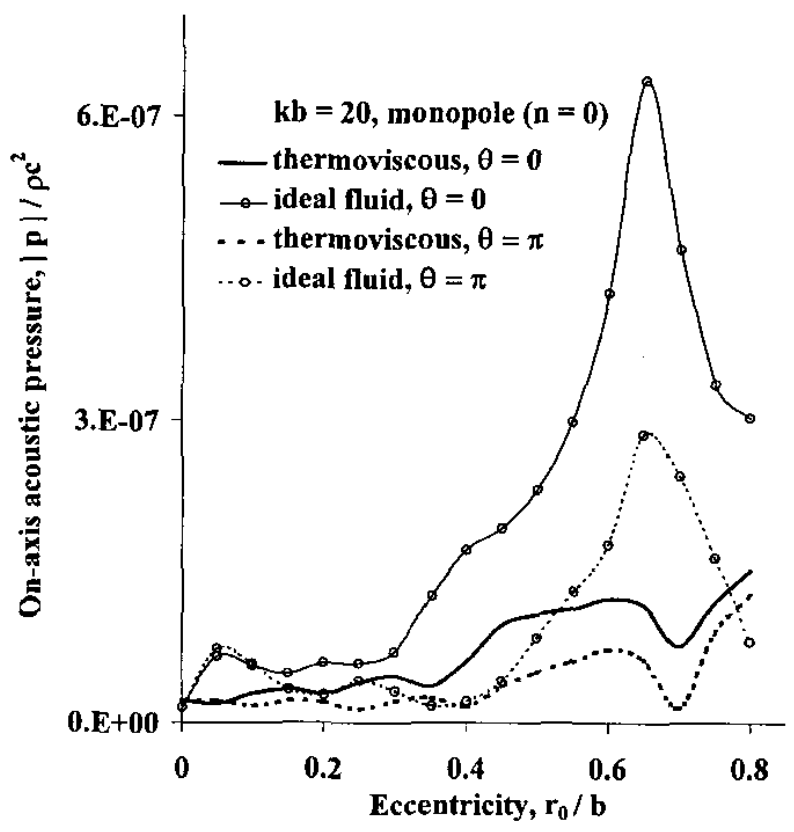

Fig. 8. Influence of source eccentricity on the on-axis radiated farfield acoustic pressures for the source vibrating in $\mathbf{n}=0$ mode at $\mathrm{kb}=20$. 


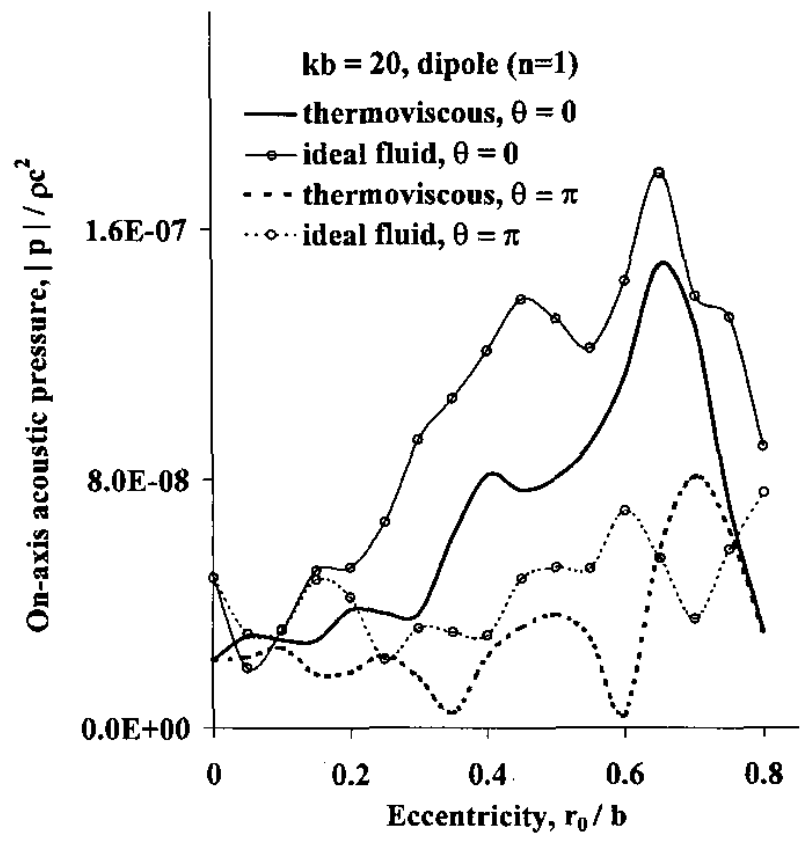

Fig. 9. Influence of source eccentricity on the on-axis radiated farfield acoustic pressures for the source vibrating in $n=1$ mode at $\mathrm{kb}=20$.

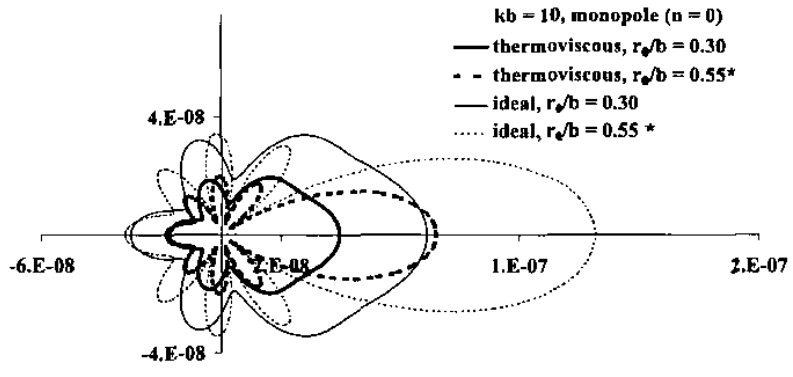

Fig. 10. Angular distribution of the radiated far-field pressure for the monopole source at selected focal points and $\mathrm{kb}=10$.

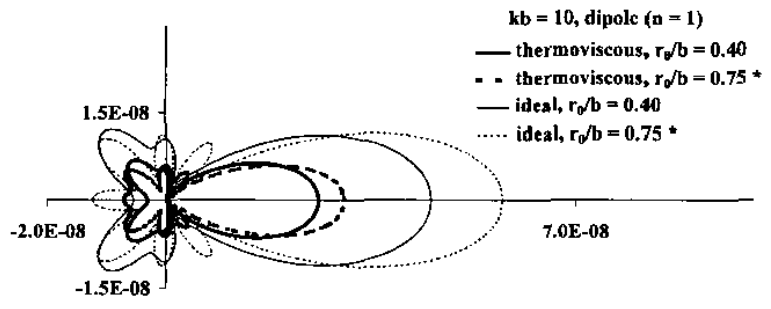

Fig. 11. Angular distribution of the radiated far-field pressure for the dipole source at selected focal points and $\mathrm{kb}=10$.

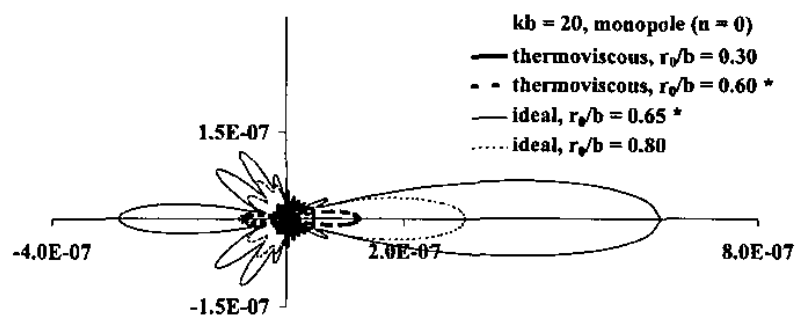

Fig. 12. Angular distribution of the radiated far-field pressure for the monopole source at selected focal points and $\mathrm{kb}=20$.

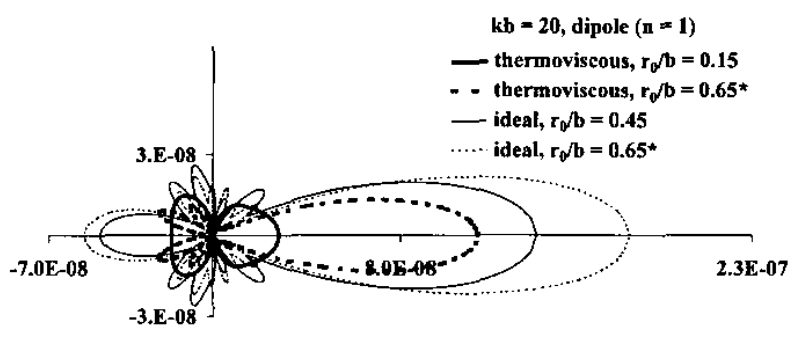

Fig. 13. Angular distribution of the radiated far-field pressure for the dipole source at selected focal points and $\mathbf{k b}=20$.

focal point. At $\mathrm{kb}=10$, thermoviscous pressure patterns have characteristically lower magnitudes but they are very similar in shape to the corresponding ideal fluid patterns. When the dimensionless wave number is doubled (i.e., for $k b=20$ ), the directionality of the radiated waves enhances and sharper (lateral) side lobes begin to appear, especially in the ideal fluid case. We also observe that, as the source is moved to the left along the negative $\mathrm{z}$-axis, forward radiation (along positive z-axis) generally dominates in both thermoviscous and ideal fluid mediums. And, to show overall validity of the work, we take advantage of the thin boundary layer concept [21] by increasing the size of the source and the fluid sphere about 100 times and executing our main (thermoviscous) code with the same input data used in Figs. 6 and 8 of [12]. Excellent agreements were obtained, as displayed in Fig. 14. Here we note that each curve is normalized to its corresponding value when the source is in an infinite medium; therefore, this impedance is denoted as relative impedance.

\section{CONCLUSIONS}

An exact study on radiation of an acoustic field due to modal vibrations of a spherical source, eccentrically positioned within a thermoviscous fluid sphere, into an external boundless viscous thermally conducting fluid medium is offered. The solution of the problem is systematically obtained by making use of the appropriate translational addition theorem in imposing the pertinent boundary conditions. The numerical results reveal the important effects of excitation frequency, mode of vibration, and eccentricity of the source on the modal impedances and the radiated far- 


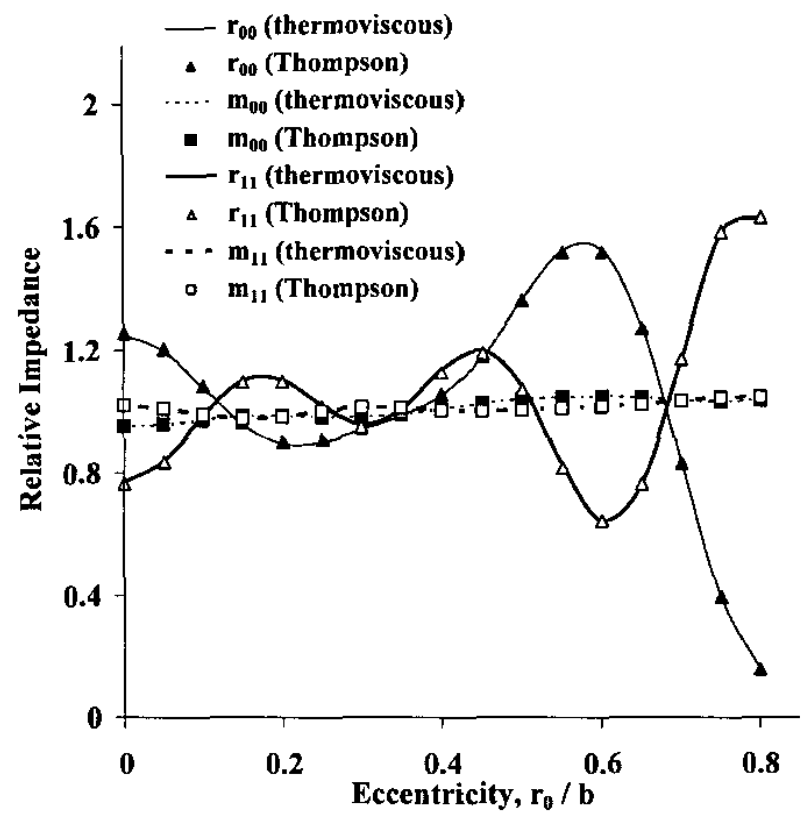

Fig. 14. Variation of relative impedance with source position at $\mathrm{kb}=11.5\left(\rho c / \rho^{*} c^{*}=0.71, c / c^{*}=0.41\right.$; monopole: $\mathrm{a} / \mathrm{b}=0.01$; dipole: $\mathrm{a} / \mathrm{b}=0.05$ )

field pressures. They also demonstrate the central role of fluid thermoviscosity in the general increase of (reduction in) the modal impedance component magnitudes (the onaxis radiated far-field acoustic pressure amplitudes). The presented exact solution can serve as the benchmark for comparison to other solutions obtained by strictly numerical or asymptotic approaches.

\section{ACKNOWLEDGMENTS}

The authors wish to sincerely thank Professors J. A. Roumeliotis and William Thompson, Jr., for valuable consultations on translational addition theorems for spherical wave functions. They also greatly appreciate the head of Iran University of Science and Technology mechanical engineering department, Professor H. Jahed Motlagh, for providing exclusive access to the computing facilities of the computational mechanics laboratory.

\section{REFERENCES}

[1] X. Zheng and R. E. Apfel, "Acoustic interaction forces between two fluid spheres in an acoustic field," J. Acoust. Soc. Amer., vol. 97 , no. 4 , pp. $2218-2226,1995$.

[2] G. C. Gaunaurd, H. Huang, and H. C. Strifors, "Acoustic scattering by a pair of spheres," J. Acoust. Soc. Amer., vol. 98, no. 1, pp. 495-507, 1995.

[3] H. Huang and G. C. Gaunaurd, "Acoustic scattering of a plane wave by two spherical elastic shells above the coincidence frequency," J. Acoust. Soc. Amer., vol. 101, no. 5, pp. 2659-2668, 1997.
[4] G. C. Gaunaurd and H. Huang, "Sound scattering by a spherical object near a hard flat bottom," IEEE Trans. Ultrason. Ferroelect., Freq. Contr., vol. 43, no. 4, pp. 690-700, 1996.

[5] H. Huang and G. C. Gaunaurd, "Scattering of a plane acoustic wave by a spherical elastic shell near a free surface," Int. $J$. Solids Struct., vol. 34, no. 5, pp. 591-602, 1997.

[6] - "Acoustic point source scattering by a spherical elastic shell submerged beneath a free surface," J. Acoust. Soc. Amer., vol. 99 , no. 5, pp. 2720-2726, 1996.

[7] G. C. Gaunaurd and H. Huang, "Acoustic scattering by an airbubble near the sea surface," IEEE J. Oceanic Eng., vol. 20, no. 4, pp. 285-292, 1995.

[8] P. Gabrielli and M. Mercier-Finidori, "Acoustic scattering by two spheres: Multiple scattering and symmetry considerations," J. Sound Vib., vol. 241, no. 3, pp. 423-439, 2001.

[9] J. A. Roumeliotis, J. D. Kanellopoulos, and J. G. Fikioris "Acoustic resonance frequency shifts in a spherical cavity with an eccentric inner small sphere," J. Acoust. Soc. Amer., vol. 90 , no. 2, pp. 1144-1148, 1991.

[10] J. A. Roumeliotis and J. D. Kanellopoulos, "Acoustic eigenfrequencies and modes in a soft-walled spherical cavity with an eccentric inner small sphere," J. Franklin Inst., vol. 329, no. 4 pp. 727-735, 1992

[11] S. M. Hasheminejad, "Modal impedances for a spherical source in a fluid-filled spherical cavity embedded within a fluidinfiltrated elastic porous medium," Int. J. Solids Struct., vol. 35 , no. $1-2$, pp. $129-148,1998$.

[12] W. Thompson, Jr., "Acoustic radiation from a spherical source embedded eccentrically within a fluid sphere," J. Acoust. Soc. Amer., vol. 54, no. 6, pp. 1694-1707, 1973.

[13] S. A. Lease and W. Thompson, Jr., "Use of translational addition theorems for spherical wave functions in nonaxisymmetric acoustic field problems," J. Acoust. Soc. Amer., vol. 90, no. 2, pt. 1, pp. 1155-1160, 1991.

[14] - "Nonaxisymmetric acoustic radiation from a pair of sources embedded in a fluid sphere," $J$. Acoust. Soc. Amer. vol. 90 , no. 2 , pt. 1 , pp. 1161-1166, 1991

[15] A. S. Dukhin, P. J. Goetz, T. H. Wines, and P. Somasundaran, "Acoustic and electroacoustic spectroscopy," Colloids Surfaces, vol. 173 , pp. $127-158,2000$.

[16] S. Temkin, "Attenuation and dispersion of sound in dilute suspensions of spherical particles," J. Acoust. Soc. Amer., vol. 108, no. 1, pp. $126-146,2000$.

[17] P. S. Epstein and J. Carhart, "The absorption of sound in suspensions and emulsions," J. Acoust. Soc. Amer., vol. 25, no. 3, pp. $553-565,1953$.

[18] J. R. Allegra and S. A. Hawley, "Attenuation of sound in suspensions and emulsions: Theory and experiments," $J$. Acoust. Soc. Amer., vol. 51, pp. 1545-1564, 1972.

[19] W. H. Lin and A. C. Rapits, "Thermoviscous effects on acous tic scattering by thermoelastic solid cylinders and spheres," $J$. Acoust. Soc. Amer., vol. 74, pp. 1542-1554, 1983.

[20] L. W. Anson and R. C. Chivers, "Ultrasonic scattering from spherical shells including viscous and thermal effects," J. Acoust. Soc. Amer, vol. 93, no. 4, pp. 1687-1699, 1993.

[21] M. Hasheminejad and T. L. Geers, "Modal impedance for two spheres in a thermoviscous fluid," J. Acoust. Soc. Amer., vol. 94, no. 4, pp. 2205-2214, 1993.

[22] W. M. Beltman, "Viscothermal wave propagation including acousto-elastic interaction," Ph.D. dissertation, University of Twente, 1998.

[23] J. D. Achenbach, Wave Propagation in Elastic Solids. New York: Elsevier North-Holland, 1976.

[24] A. D. Pierce, Acoustics; An Introduction to Its Physical Principles and Applications. New York: Acoustical Society of America, 1991 .

[25] A. C. Eringen, Mechanics of Continua. New York: Krienger, 1980.

[26] M. Abramowitz and 1. A. Stegun, Handbook of Mathematical Functions. Washington, DC: National Bureau of Standards, 1965.

[27] Y. A. Ivanov, Diffraction of Electromagnetic Waves on Two Bodies. Minsk: Nauka i Tekhnika, 1968; NASA Tech. Translation F-597, 1970 .

[28] M. C. Junger and D. Feit, Sound, Structures, and Their Interaction. 2nd ed. Cambridge, MA: MIT Press, 1986.

[29] On the acoustic properties for 3M FC., http://www.mmm.com. 
[30] N. B. Vargaftik, Handbook of Physical Properties of Liquids and Gases. Berlin: Springer-Verlag, 1983.

[31] W. H. Press, B. P. Flannery, S. A. Teukolsky, and W. T. Vetterling, Numerical Recipes. Cambridge, U.K.: Cambridge Univ. Press, 1987.

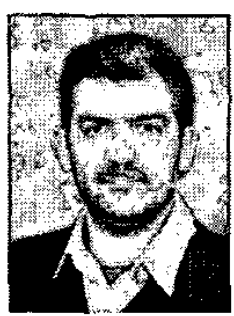

Seyyed M. Hasheminejad was borne in Tehran, Iran, on July 6, 1962 . He received the B.S. degree in mechanical engineering in 1983 from California State University, Chico, CA; the M.S. degree in mechanical engineering in 1985 from Santa Clara University, Santa Clara, CA; and the Ph.D. degree in mechanical engineering in 1992 from the University of Colorado, Boulder, CO.

Since 1993, he has been an assistant professor at the mechanical engineering department of Iran University of Science and Technology, Tehran, Iran. He has also been a part-time lecturer at Research Division of Azad University, Tehran, Iran, since 1999. He has conducted (consulted in) various national research projects, including design and construction of high-power underwater piezoelectric transducers, automotive acoustic silencers, sound absorbers (acoustic foams) and insulators, vibration damping treatments, pneumatic mounts, broadcasting studios; and noise and vibration control in automobiles, airplanes, trains, residential and industrial environments. He is currently involved in development of national standards, specifications and guidelines on environmental noise and vibration control.

Dr. Hasheminejad is a member of various professional societies in Iran such as the Iranian Society of Mechanical Engineers, the Iranian Society of Electrical Engineers, and the Iranian Society of Aerospace Engineers. He is an active reviewer for various journals and evaluating boards, and a member of several technical committees in professional societies in Iran.

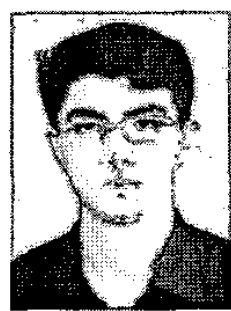

Mahdi Azarpeyvand was borne in Tehran, Iran, in 1981 . He was among the top student entries through the National Iranian Higher Education Exam. Since 1999 he has been working at Iran University of Science and Technology toward the B.S. degree in mechanical engineering. His research interests include scattering, wave propagation, and boundary value problems in electromagnetics and acoustics. He is a member of various student societies such as the student branch of the Iranian Society of Mechanical Engineers, and the Iranian Physics Society. He has received several awards as an outstanding student researcher. 